\title{
Treatment of malignant pleural mesothelioma: current status and future directions
}

\author{
X. Dhalluin 1,2, A. Scherpereel1,2,3
}

\begin{abstract}
Treatment of malignant pleural mesothelioma: current status and future directions. X. Dhalluin, A. Scherpereel.

Previously considered to be rare, malignant pleural mesothelioma (MPM) is a highly aggressive tumour that has become a very important issue over recent years due to its poor prognosis and its increasing incidence mostly linked to previous asbestos exposure. An optimal treatment for MPM is not established yet; new therapies and predictive tools are still needed in the management of this cancer.
\end{abstract}

\begin{abstract}
Thus the aim of this review is to provide clinicians clear and up-to-dated data on the latest therapeutic strategies for MPM patients in 2010. The guidelines recently proposed by the European Respiratory Society (ERS) and the European Society of Thoracic Surgeons (ESTS) taskforce are summarized here. The authors also briefly reviewed the future directions in MPM treatment including targeted therapies, gene or cell therapies.
\end{abstract}

Monaldi Arch Chest Dis 2010; 73: 2, 79-85.

\footnotetext{
1 Pneumologie et Oncologie Thoracique, Hôpital Calmette - CHRU de Lille, Lille Cedex,

2 University of Lille II, Medical School Henri Warembourg, Lille,

3 Unit INSERM 1019, CIIL, Institut Pasteur de Lille, France.
}

Correspondence: Arnaud Scherpereel; Pneumologie et Oncologie Thoracique, Hôpital Calmette - CHRU de Lille, 59037 Lille Cedex, France; e-mail: arnaud.scherpereel@chru-lille.fr

\section{Introduction}

Malignant pleural mesothelioma (MPM) is a rare but highly aggressive tumor with poor prognosis and an increasing incidence. An optimal treatment of MPM is not clearly defined yet, even if clear and up-to-date guidelines on management of MPM patients were recently proposed by the European Respiratory Society (ERS) in collaboration with the European Society of Thoracic Surgeons (ESTS) [1]. These guidelines and an overview of potential future therapies for this cancer are summarized in this review.

\section{Current status}

\section{Surgery for MPM}

Main surgical procedures for MPM are pleurectomy/decortication (P/D, debulking surgery), a partial macroscopic clearance of tumour performed by thoracotomy or by video assisted thoracoscopic surgery (VATS), and extrapleural pneumonectomy (EPP) considered as "radical" surgery. There is very limited evidence supporting P/D but VATS may provide good symptom control and have an impact on survival [2]. An ongoing study in United Kingdom is com- paring VATS debulking with chemical pleurodesis (MesoVATS). Thus P/D should not be proposed in a curative intent but rather to symptomatic (breathless, pain) patients with entrapped lung syndrome who cannot benefit from talc pleurodesis (grade of recommendation: $2 \mathrm{C}$ ). Pleural undwelled catheters are also an option in this case.

There is also limited data supporting "radical" surgery (EPP) for mesothelioma, and only for EPP as part of multimodal treatment [3-5]. Median survival of patients benefiting from such strategy was 18-24 months. EPP mortality is about 5\% in experienced centres [5] while morbidity is up to $50 \%$. Therefore it is recommended that EPP should be performed only in clinical trials, in specialized centres, as part of multimodal treatment [1].

\section{Radiotherapy $(R T)$ in MPM}

\section{POST-OPERATIVE RADIOTHERAPY}

As evidence were limited and came from retrospective studies, the ERS/ESTS experts recommended that RT should not be performed after P/D (1A).

Recurrence rate of post-EPP RT may vary from $13 \%$ to $50 \%$, and local-only recurrence rate from $4 \%$ to $13 \%[4,6]$. RT technical issues may partly explain these discrepancies. This procedure 
should only be realized in clinical trials in dedicated centres (1A). A randomized European (SAKK) study is ongoing to assess the efficacy and tolerance of post-EPP RT.

In fact large fields, complex target shape and proximity of organs at risk such may limit dose and efficacy of RT in MPM. Intensity-modulated radiotherapy (IMRT) seems to be a relevant alternative as it theoretically allows large irradiations of complex fields. Preliminary results of IMRT after EPP were promising but several cases of fatal pneumonitis have been described [7-10]. Therefore, post-operative RT, including IMRT, should be performed only in expert centres, as part of clinical trials [1].

\section{PALLIATIVE OR PROPHYLACTIC RADIOTHERAPY}

In case of painful chest wall infiltration or nodules, palliative RT may be a useful treatment [1].

An irradiation with $7 \mathrm{~Gy} /$ day for three consecutive days within four weeks after any pleural procedure has been established to prevent subcutaneous tumour seeding developing along drainage channels or thoracocentis tracts [11]. However, a small recent randomised trial as well as the Cochrane Overview [12] did not support prophylactic RT. There was no difference for tract tumour seeding between the RT arm and the no-RT arm $[13,14]$. Underpowered studies and suboptimal irradiation techniques may explain the inconsistency of these results. Thus, as the value of prophylactic RT is questionable, the ERS/ESTS experts were not able to propose any firm recommendation [1]. However, based on previous French Pulmonary Society guidelines $[15,16]$ and our own centre experience, we decided to continue to use prophylactic RT, a cheap, easy and efficient procedure till a new significant study on this issue.

\section{Chemotherapy (CT) of MPM}

Significant improvement in MPM management was obtained by the first two large, multicentric, prospective and randomised phase III trials $[17,18]$. Previous trials were often little informative because they usually reported small series of patients in monocentric, non-randomised and/or retrospective studies $[1,19]$.

\section{FIRST-LINE CT IN MPM: DOES IT WORTH?} WHICH DRUG(S) TO BE USED?

Only one randomised study assessed the value of $\mathrm{CT}$ versus placebo in addition to best supportive care (BSC) [20]. No significant difference for survival was found between the two arms. However this trial had several major limits because it was prematurely stopped due to a poor recruitment, and the drugs were not the optimal ones in this indication. In fact, phase III randomised trials $[17,18]$ suggested that cisplatin combined with antifolate, i.e. pemetrexed (C/P, with supplementation by folic acid and B12 vitamin) or raltitrexed $(\mathrm{C} / \mathrm{R})$, could increase response rate (RR) and median survival time (MST) compared to cisplatin alone (C/P: 12.1 months or C/R: 11.4 months vs 9.3 and 8.8 months for cisplatin alone arms, respectively). Therefore combination of platinum and pemetrexed or raltitrexed is recommended as standard first line chemotherapy for patients with good performance status (PS<3) (1B) [1].

\section{OPTIMAL TIMING TO START FIRST LINE \\ CT - DURATION OF TREATMENT}

Some indirect evidences suggest starting CT immediately after the diagnosis. First, an initial adequate PS was predictive of a better survival for patients in phase III trials [17, 18]; and large tumors may be less sensitive to CT [21-23]. Moreover results of a small randomised trial supported immediate first line CT versus delayed treatment at the time of symptoms progression, with a time to symptomatic progression ( 25 versus 11 weeks, respectively) and MST (14 versus 10 months; 1 -year $66 \%$ versus $36 \%$, respectively), although the difference was not statistically significant $(p=0,1)$ [24].

There are no data suggesting delivering more than 6 cycles in stable or responding patients (2C) or supporting maintenance treatment with $\mathrm{CT}$ or targeted therapies. CT should also be stopped in case of progressive disease, grade 3-4 toxicities, or cumulative toxic doses (1A) [1].

There were a few studies evaluating the value of intrapleural therapies in MPM. To date, this treatment exhibited limited efficiency and high toxicity. Therefore it should be only considered in clinical trials [25].

\section{SECOND-LINE TREATMENT}

Second line treatment seems a reasonable issue as many patients having progressive disease still fit to benefit from another treatment. Thus, in a retrospective analysis of study of Vogelzang et al, $42 \%$ of the patients received second line treatment [26]. Moreover post-study treatment (PST) was associated with a better survival whatever cytotoxic drugs were used.

An extensive review of the literature concluded that no drug or combination of drugs have been validated in second line CT [1]. However, the pemetrexed expanded access programm (EAP) provided interesting data in this setting [27]. The treatment was well tolerated in 187 patients receiving pemetrexed alone ("P"; $\mathrm{n}=91$ ) or in association with cisplatin ("C/P"; $n=96$ ). No direct comparison was done beween the different treatments. But in 80 "C/P" vs 73 "P" assessable patients, RR was $32.5 \%$ vs $5.5 \%$, disease control rate was $68.8 \%$ vs $41 \%$, and overall survival (OS) was 7.1 months (95\% CI; 6.5-11) vs 4.1 months $(95 \%$ CI; 3.2- N/A), respectively. Moreover, in a phase III randomised trial comparing pemetrexed and BSC versus $\mathrm{BSC}$ alone, there was a benefit in the CT arm in terms of survival without disease progression, RR and time to progression (TTP) but not for OS [28]. Vinorelbine might also be a reasonable choice in second line treatment and has recently exhibited on a series of 63 patients a $16 \%$ RR and MST of 9.6 months in this setting [29]. To date, it is recommended that patients demonstrating pro- 
longed symptomatic and objective response with first line CT may be treated again with the same regimen in the event of recurrence (2C). Other patients, in a good PS, should rather be proposed to participate to clinical trials (2C) [1].

\section{TARGETED THERAPIES AND OTHER BIOTHERAPIES IN MPM}

Immunomodulators such as interferons or interleukin-2 seemed not effective alone and are not recommended outside of a clinical trial. Preliminary results of Mycobacterium vaccae administration seemed interesting but need confirmation. Ranpirnase has not demonstrated its effectiveness in trials. Several targeted therapies were also tested in malignant mesothelioma but no positive clinical trial was published yet. Main drugs and results are summarized in the second part of the review. To date, immunomodulating agents, targeted treatments and other biotherapies should not be used in the treatment of MPM outside clinical trials (1C) [1].

\section{WHAT ASSESSMENT CRITERIA SHOULD BE USED} FOR PATIENTS MONITORING IN MPM?

The response to the treatment can be assessed on [1]:

- clinical criteria such as symptoms control and quality of life (modified version of the Lung Cancer Symptom Scale (LCSS) recommended) [30];

- imaging criteria: CT-scan is recommended for assessment and follow-up of MPM. If a patient has had pleurodesis, chest CT-scan should be performed again before starting chemotherapy in order to better evaluate the response to treatment (1B). The modified RECIST criteria [31-33] are the preferred method of measuring response to treatment (1B). Positron emission tomography (PET) combined CT-scan may allow a better response evaluation but is still not validated in routine in MPM;

- $\quad$ survival parameters (OS rather than TTP);

- biological markers are still under investigation for patients monitoring in MPM. However, serum mesothelin seems to be a promising biomarker according to recent reports and was already approved in the USA by the FDA for compassional use [34, 35];

- reassessment of MPM by thoracoscopy was never published.

\section{Multimodal strategy}

As surgery alone is not curative in MPM because resection margins can be hardly obtained, it has been proposed to combine radiotherapy and/or chemotherapy to surgery. However, this strategy may improve the treatment efficacy but also cumulate specific toxicities. Therefore the value multimodal approach is still highly debated and patients who are potential candidates for this procedure need to previously undergo a thorough work-up. This work-up should include at least physical examination, pulmonary function tests and cardiac function assessment, and imaging procedures for tumour staging [1].
The following criteria are usually considered for potential EPP and multimodal treatment: histological proof of non-sarcomatoid MPM subtype, clinical and/or pathological stage T1-3, N0-1 ( $\leq \mathrm{N} 2$ for a few centres), M0 patient fit to have pneumonectomy and to receive neoadjuvant/adjuvant chemotherapy and adjuvant radical hemithorax irradiation [1].

There are limited and weak data available on the best combination treatment, and only recent studies included optimal chemotherapy. Morever, multimodal approach including EPP is usually responsible for morbidity up to $70 \%$ and mortality rate $\leq 7 \%$ in specialized centres. In a Swiss study recruiting 61 patients with $\mathrm{MPM} \leq \mathrm{cT} 3 \mathrm{~N} 2 \mathrm{M} 0$ treated by 3 cycles of cisplatin-gemcitabine followed by EPP and limited radiation to high-risk sites, only 45 patients benefited from full treatment and exhibited a MST of 23 months [5]. The European phase II trial EORTC 08031 assessed in 58 patients with MPM $\leq \mathrm{cT} 3 \mathrm{~N} 1 \mathrm{M} 0$ the feasibility of induction CT ( 3 cycles platinum-pemetrexed) followed in non-progressing subjects by EPP and postoperative RT (54 Gy, 30 fractions) [36]. Primary endpoint was "success of treatment" meaning that patients received full treating without grade 3-4 toxicity and did not progressed within 90 days post treatment. Only 24 patients $(42 \%)$ met these criteria (one-sided 90\% CI 0.36-1.00), having median OS of 18.4 months (95\% CI 15.6-32.9) and PFS of 13.9 months (95\% CI 10.9-17.2).

Finally the Mesothelioma and Radical Surgery (MARS) controlled trial randomized patients after platinum-based CT, if still eligible, to surgery (EPP) followed by radical hemithorax RT versus no surgery [37]. Only 50 patients out of 112 (45\%) patients entering the evaluation and induction phase of the trial went on to be randomized. Thus randomization between surgery and no surgery was feasible but results were quite deceptive as previous studies. Therefore, as this approach is still not validated, that patients who are considered candidates for this multimodal strategy should be included in prospective randomised trials in dedicated centres [1].

\section{Future directions for systemic treatment of MPM}

New pathways identified in MPM pathogenesis led to new targets and innovative therapies. These targeted therapies and biotherapies, still under investigation, are summarized below.

\section{Epidermal Growth Factor Receptor (EGFR)}

The tyrosine kinase (TK) EGF receptor pathway is involved in angiogenesis, proliferation, survival, and migration of tumor cells, and is over-expressed in MPM. Govindan et al evaluated the efficacy of the EGFR TK inhibitor gefitinib in 43 non-selected MPM patients for activating EGFR mutation [38]. The RR was low: one partial response (PR), one complete response (CR) and 21 stable disease (SD). Similar deceptive results were found with erlotinib in 63 unselected chemonaive 
patients [39]. No objective response was observed if toxicity was acceptable.

\section{Vascular endothelial growth factor (VEGF) inhibitors and other anti-angiogenic drugs}

VEGF is an autocrine growth factor having a key role in tumor angiogenesis and lymphangiogenesis in mesothelioma [40, 41]. Moreover VEGF and VEGF receptors are highly expressed in MPM. Several anti-angiogenic drugs were tested alone or in combination with other agents in MPM.

In a phase II trial, no objective response was observed in 24 MPM patients treated by antiVEGF antibodies bevacizumab (15 $\mathrm{mg} / \mathrm{kg} / 3$ weeks) in combination with erlotinib $150 \mathrm{mg}$ /day [42]. In a randomised phase II trial assessing cisplatin-gemcitabine with or without bevacizumab, the addition of bevacizumab did not result in improved response rate (25\% versus $22 \%$ ) nor survival rate (MST 15.6 months vs 14.7 months; $\mathrm{p}=0.91$ ) [43]. This may be partly due to second line $\mathrm{CT}$ by pemetrexed resulting in a response rate of $12.1 \%$ and SD in $46 \%$ of pre-treated patients, in one year survival rate of $54 \%$ and in median time to progression of 4.9 months. Interestingly, low initial serum VEGF level was associated with a better overall survival and PFS in all patients $(\mathrm{p}=0.008)$. A French phase II randomised clinical trial "MAPS", evaluating first line CT combining pemetrexed-cisplatin $(\mathrm{PC}) \pm$ bevacizumab $(\mathrm{PCB})$ in 111 patients, was just achieved on January 2010. Preliminary promising results were presented during 2010 ASCO meeting [44]. The PCB chemotherapy was well-tolerated and feasible. In the first 93 eligible patients, there were $27 / 47$ patients $(57.4 \%)$ with disease control (1 CR, 17 PR, 9 stable disease) in the PCB Arm vs. 21/46 $(45.7 \%)$ in the PC arm $(\mathrm{p}=0.01)$.

Thalidomide, another anti-angiogenic drug through inhibition of VEGF, bFGF, and TGF- $\alpha$, was tested alone or in combination with chemotherapy $[45,46]$. In a phase I/II trial testing thalidomide alone [46], there was no objective response but 11 patients/40 (27\%) had a SD more than 6 months; MST was 11 months; TTP was 8 weeks. A smaller Australian study $(n=22)$ had similar results. Finally, in 16 chemonaive patients treated by cisplatin, gemcitabine and thalidomide, PR and SD rates were $14 \%$ and $55 \%$, respectively; MST was 12 months; TTP was 17 weeks. Other trials with thalidomide are ongoing.

There are very limited clinical data on multitargets (TK) drugs such as sorafenib, vatalanib, pazopanib, or sunitinib in MPM. Although some patients experienced objective response, endpoints of the different studies were never achieved, and new trials are ongoing. Phase II trials assessing imatinib mesylate in MPM were deceptive with no objective response (SD: 12-44\%) [47-49]. VEGFR-2 inhibitor semaxanib seemed to be efficient (response rate 11\%; MST 12.3 months) but was associated with an intolerable risk of thrombosis [50].
A phase II trial evaluated tetrathiomolybdate (TM), a potential anti-angiogenic and anti-VEGF drug through copper depletion and ceruleoplasmin decrease, starting 4 to 6 weeks after debulking surgery in 30 stage I-III patients compared to a historic series of 164 patients from the same group [51]. Toxicity was low and TM exhibited a potential value in stages I-II disease only, but methodological issues did not permit any firm conclusion.

\section{Ribonuclease inhibitors}

Ranpirnase is an enzyme degrading tRNA in the Golgi system resulting in inhibition of proteins synthesis including proteins involved in cell replication and apoptosis. The role of ranpirnase in MPM, alone or in combination with doxorubicin, was evaluated through phases II and III trials with deceptive results [1].

\section{Histone deacetylase inhibitors (HDACi)}

HDAC are a large group of $\mathrm{Zn}^{2+}$-dependent enzymes are potential targets in cancer because inhibition of histone deacetylation results, through acetylation of histone proteins, in expression of genes associated to cell cycle arrest, apoptosis and tumor suppression. Moreover HDACi lead to acetylation of nonhistone proteins leading to other anticancer effects such as inhibition of angiogenesis, motility and invasion of tumor cells [52]. Many class-specific or pan HDACi have been tested in different cancers. For example, HDACi such as vorinostat (SAHA), panobinostat or valproic acid (VPA) are evaluated in lung cancer patients in combination with chemotherapy [53-55]. Vorinostat is already FDA-approved in the treatment of cutaneous T-cell lymphoma

Vorinostat was tested in previously treated MPM patients. In a phase I study $(n=13)$, it appeared to be well-tolerated [56]. Then in a phase II, vorinostat was evaluated versus placebo. Despite unpublished results of this phase II study, a large randomised controlled phase III trial with the same design is ongoing.

Belinostat, a novel HDACi, was also tested in 13 MPM patients including 11 patients previously treated by pemetrexed-platinum [55]. The study was stopped because belinostat was considered as inefficient single agent: no objective response was found; MST was 1 month and OS was 5 months.

In a recent phase II study, the value and the safety of valproic acid (VPA) combined with doxorubicin was assessed in 45 MPM patients (PS 02) after at least one chemotherapy line [57]. RR was $16 \%$ (95\% CI 3-25\%), disease control rate was $36 \%$ (95\% CI 22-51\%); OS was 6.7 months (95\% CI 4.9-8.5 months). Toxicity was acceptable as severe neurological toxicity was seen, main toxicity was leuco-neutropenia induced by doxorubicin.

This was the first phase II suggesting clearly an anti-tumor effect of HDACi in mesothelioma, associated with an improvement of survival. 


\section{Immunomodulators}

Interferons and interleukins were tested in MPM with some success. But doses, method of administration (intrapleural or systemic), drugs and disease stage varied highly from one study to another. Therefore these results must be cautiously analyzed and, to date, monotherapy with interferons or interleukin-2 seemed not effective and are not recommended outside of a clinical trial [1].

Interesting preliminary results were observed after administration of Mycobacterium vaccae in a limited number of patients. This needs to be confirmed before recommend the use of this treatment.

Gene therapy has shown promising results in mesothelioma in pre-clinical models Anti-tumor response in MPM was demonstrated in murine models and in patients during phase I trial when injecting intra-pleurally adenoviral vectors (Ad) with thymidine kinase gene (associated with ganciclovir IV) or IFN- $\beta$ gene $[58,59]$. CD $4+$ and CD8+ tumor-specific T cells appeared as key effector cells for tumor inhibition [60], suggesting a potential benefit to associate cell therapy to this strategy. Significant tumor inhibition was also shown in animal models, but not in humans so far, by transfection of p53, Bak, p14arf, or CD40 ligand genes, or using antisens oligonucleotides (ODN) to block the expression of some genes such as growth factors (PDGF $\alpha$ et $\beta$, IGF I, TGF- $\beta \ldots$..) $[61,62]$.

Cell therapy seems to be another interesting treatment in MPM [63-65]. In fact, if chemotherapy may increase RR to treatment and survival in non-resectable patients, there are always some resistant tumor cells that may inactive the immune system. Thus it is necessary to stimulate and to "educate" antigen presenting cells (dendritic cells...) and immune effector cells (natural killer (NK) cells, cytotoxic T cells) to eliminate these tumor cells. Associated to other standard therapies (chemotherapy...), this vaccine strategy may improve the treatment of MPM patients. Encouraging results were recently published of a phase I trial assessing in 10 MPM patients the safety and immunological response induced by the intradermally and intravenously administration of tumor lysate-pulsed dendritic cells (DC) at two-week intervals after CT [65]. No grade 3 or 4 toxicities or any evidence of autoimmunity were observed. Interestingly, local accumulations of infiltrating $\mathrm{T}$ cells were found at the site of vaccination. Immunological response to tumor cells was detected in a subgroup of mesothelioma patients.

\section{Conclusion}

Significant improvement has been achieved recently in the management of MPM, reflected by the guidelines from European scientific societies [1]. However many questions about MPM treatment, in particular about second line treatment and the role of targeted therapies, remain without clear and validated answer. Moreover the overall sur- vival of mesothelioma patients is still poor. These facts should stimulate clinicians to recruit these patients as much as possible in prospective and randomised clinical trials to improve the treatment of mesothelioma.

\section{References}

1. Scherpereel A, Astoul P, Baas P, Berghmans T, et al. European Respiratory Society/European Society of Thoracic Surgeons Task Force. Guidelines of the European Respiratory Society and the European Society of Thoracic Surgeons for the management of malignant pleural mesothelioma. Eur Respir J 2010; 35: 479-95.

2. Halstead JC, Lim E, Venkateswaran RM, Charman SC, Goddard M, Ritchie AJ. Improved survival with VATS pleurectomy-decortication in advanced malignant mesothelioma. Eur J Surg Oncol 2005; 31: 314-20.

3. Sugarbaker DJ, Jaklitsch MT, Bueno R, et al. Prevention, early detection, and management of complications after 328 consecutive extrapleural pneumonectomies. $J$ Thorac Cardiovasc Surg 2004; 128: 138-46.

4. Rusch VW, Rosenzweig K, Venkatraman E, et al. A phase II trial of surgical resection and adjuvant highdose hemithoracic radiation for malignant pleural mesothelioma. J Thorac Cardiovasc Surg 2001; 122: 788-95.

5. Weder W, Stahel RA, Bernhard J, et al. Multicenter trial of neo-adjuvant chemotherapy followed by extrapleural pneumonectomy in malignant pleural mesothelioma. Ann Oncol 2007; 18: 1196-202.

6. Baldini EH. External beam radiation therapy for the treatment of pleural mesothelioma. Thorac Surg Clin 2004; 14: 543-8.

7. Allen AM, Czerminska M, Janne PA, et al. Fatal pneumonitis associated with intensity-modulated radiation therapy for mesothelioma. Int J Radiat Oncol Biol Phys 2006; 65: 640-5.

8. Rice DC, Stevens CW, et al. Outcomes after extrapleural pneumonectomy and intensity-modulated radiation therapy for malignant pleural mesothelioma. Ann Thorac Surg 2007; 84: 1685-92; discussion 1692-3.

9. Kristensen CA, Nottrup TJ, et al. Pulmonary toxicity following IMRT after extrapleural pneumonectomy for malignant pleural mesothelioma. Radiother Oncol 2009; 92: 96-9.

10. Miles EF, Larrier NA, et al. Intensity-modulated radiotherapy for resected mesothelioma: the Duke experience. Int J Radiat Oncol Biol Phys 2008; 71: 1143-50.

11. Boutin C, Rey F, Viallat JR. Prevention of malignant seeding after invasive diagnostic procedures in patients with pleural mesothelioma. A randomized trial of local radiotherapy. Chest 1995; 108: 754-8.

12. Chapman E BE, Diéguez M, Ortiz Z. Radiotherapy for malignant pleural mesothelioma. Cochrane Database of Systematic Reviews 2006.

13. O'Rourke N, Garcia JC, Paul J, Lawless C, McMenemin R, Hill J. A randomised controlled trial of intervention site radiotherapy in malignant pleural mesothelioma. Radiother Oncol 2007; 84: 18-22.

14. Muirhead R, O'Rourke N. Drain site radiotherapy in malignant pleural mesothelioma: a wasted resource. Eur Respir J 2007; 30: 1021.

15. Scherpereel A. The experts' conference of the Societe de Pneumologie de Langue Francaise (SPLF) on malignant pleural mesothelioma (MPM): useful and necessary recommendations. Rev Mal Respir 2006; 23: 11S5-6.

16. Scherpereel A. Guidelines of the French Speaking Society for Chest Medicine for management of malignant pleural mesothelioma. Respir Med 2007; 101: 1265-76. 
17. Vogelzang NJ, Rusthoven JJ, et al. Phase III study of pemetrexed in combination with cisplatin versus cisplatin alone in patients with malignant pleural mesothelioma. J Clin Oncol 2003; 21: 2636-44.

18. van Meerbeeck JP, Gaafar R, et al. Randomized phase III study of cisplatin with or without raltitrexed in patients with malignant pleural mesothelioma: an intergroup study of the European Organisation for Research and Treatment of Cancer Lung Cancer Group and the National Cancer Institute of Canada. J Clin Oncol 2005; 23: 6881-9.

19. Berghmans T, Paesmans M, et al. Activity of chemotherapy and immunotherapy on malignant mesothelioma: a systematic review of the literature with metaanalysis. Lung Cancer 2002; 38: 111-21.

20. Muers MF, Stephens RJ, Fisher P, et al. Active symptom control with or without chemotherapy in the treatment of patients with malignant pleural mesothelioma (MS01): a multicentre randomised trial. Lancet 2008; 371 (9625): 1685-94.

21. DeVita VT, Jr. The James Ewing lecture. The relationship between tumor mass and resistance to chemotherapy. Implications for surgical adjuvant treatment of cancer. Cancer 1983; 51: 1209-20.

22. Skipper HE, Schabel FM, Jr, Wilcox WS. Experimental Evaluation of Potential Anticancer Agents. Xiii. On the Criteria and Kinetics Associated with "Curability" of Experimental Leukemia. Cancer Chemother Rep 1964; 35: 1-111.

23. Skipper HE. Laboratory models: some historical perspective. Cancer Treat Rep 1986; 70: 3-7.

24. O'Brien ME, Watkins D, Ryan C, et al. A randomised trial in malignant mesothelioma (M) of early (E) versus delayed (D) chemotherapy in symptomatically stable patients: the MED trial. Ann Oncol 2006; 17: 270-5.

25. Tsao AS, Mehran R, et al. Neoadjuvant and intrapleural therapies for malignant pleural mesothelioma. Clin Lung Cancer 2009; 10: 36-41.

26. Manegold C, Symanowski J, et al. Second-line (poststudy) chemotherapy received by patients treated in the phase III trial of pemetrexed plus cisplatin versus cisplatin alone in malignant pleural mesothelioma. Ann Oncol 2005; 16: 923-7.

27. Janne PA, Wozniak AJ, et al. Pemetrexed alone or in combination with cisplatin in previously treated malignant pleural mesothelioma: outcomes from a phase IIIB expanded access program. J Thorac Oncol 2006; 1: 506-12.

28. Jassem J, Ramlau R, Santoro A, et al. Phase III trial of pemetrexed plus best supportive care compared with best supportive care in previously treated patients with advanced malignant pleural mesothelioma. J Clin Oncol 2008; 26: 1698-704.

29. Stebbing J, Powles T, McPherson K, et al. The efficacy and safety of weekly vinorelbine in relapsed malignant pleural mesothelioma. Lung Cancer 2009; 63: 94-7.

30. Hollen PJ, Gralla RJ, Liepa AM, Symanowski JT, Rusthoven JJ. Measuring quality of life in patients with pleural mesothelioma using a modified version of the Lung Cancer Symptom Scale (LCSS): psychometric properties of the LCSS-Meso. Support Care Cancer 2006; 14: 11-21.

31. van Klaveren RJ, Aerts JG, de Bruin H, Giaccone G, Manegold C, van Meerbeeck JP. Inadequacy of the RECIST criteria for response evaluation in patients with malignant pleural mesothelioma. Lung Cancer 2004; 43: 63-9.

32. Byrne MJ, Nowak AK. Modified RECIST criteria for assessment of response in malignant pleural mesothelioma. Ann Oncol 2004; 15: 257-60.

33. Nowak AK. CT, RECIST, and malignant pleural mesothelioma. Lung Cancer 2005; 49 Suppl 1: S37-40.
34. Grigoriu BD, Chahine B, Vachani A, et al. Kinetics of soluble mesothelin in patients with malignant pleural mesothelioma during treatment. Am J Respir Crit Care Med 2009 May 15; 179: 950-4.

35. Wheatley-Price P, Yang B, Patsios D, et al. Soluble Mesothelin-Related Peptide and Osteopontin As Markers of Response in Malignant Mesothelioma. J Clin Oncol 2010 May 24. [Epub ahead of print]

36. Van Schil PE, Baas P, Gaafar R, et al. on behalf of the EORTC Lung Cancer Group. Phase II trial of trimodality therapy for malignant pleural mesothelioma (EORTC 08031). Eur Respir J 2010 Jun 4. [Epub ahead of print]

37. Treasure T, Waller D, Tan C, et al. The Mesothelioma and Radical surgery randomized controlled trial: the Mars feasibility study. J Thorac Oncol 2009; 4: 1254-8.

38. Govindan R, Kratzke RA, et al. Gefitinib in patients with malignant mesothelioma: a phase II study by the Cancer and Leukemia Group B. Clin Cancer Res 2005; 11: 2300-4.

39. Garland LL, C. Rankin, et al. Phase II study of erlotinib in patients with malignant pleural mesothelioma: a Southwest Oncology Group Study. J Clin Oncol 2007; 25: 2406-13.

40. Strizzi L, Catalano A, et al. Vascular endothelial growth factor is an autocrine growth factor in human malignant mesothelioma. J Pathol 2001; 193: 468-75.

41. Ohta Y, Shridhar V, et al. VEGF and VEGF type C play an important role in angiogenesis and lymphangiogenesis in human malignant mesothelioma tumours. $\mathrm{Br} \mathrm{J}$ Cancer 1999; 81: 54-61.

42. Jackman DM, Kindler HL, et al. Erlotinib plus bevacizumab in previously treated patients with malignant pleural mesothelioma. Cancer 2008; 113: 808-14.

43. Kindler HLK, Gandara R, et al. Final analysis of a multi-center, double-blind, placebo-controlled, randomized phase II trial of gemcitabine/cisplatin (GC) plus bevacizumab $(\mathrm{B})$ or placebo $(\mathrm{P})$ in patients with malignant mesothelioma: C5-06. World lung cancer Conference 2007.

44. Zalcman G, Margery J, Scherpereel A, et al. on behalf of IFCT. IFCT-GFPC-0701 MAPS trial, a multicenter randomized phase II/III trial of pemetrexed-cisplatin with or without bevacizumab in patients with malignant pleural mesothelioma. J Clin Oncol 2010; 28: 15s (suppl; abstr 7020).

45. Pavlakis N, WG, Harvey R, Aslani A, Abraham R, Wheeler $\mathrm{H}$. Thalidomide alone or in combination with cisplatin/gemcitabine chemotherapy for malignant mesothelioma (MM): preliminary results from two phase II studies. 2002 ASCO annual meeting.

46. Baas $\mathrm{P}$, Boogerd $\mathrm{W}$, et al. Thalidomide in patients with malignant pleural mesothelioma. Lung Cancer 2005; 48: 291-6.

47. Millward M, PF, Byrne M, Powell A, Dunleavey R, Lynch K, Boyer MJ. Phase II trial of imatinib mesylate in patients with advanced pleural mesothelioma. 2003 ASCO Meeting. Proc Am Soc Clin Oncol 2003; 22: 2003 (abstr 912).

48. Villano JL, HA, Stadler WM, Hanson LL,Vogelzang NJ, Kindler HL. A phase II trial of imatinib mesylate in patients with malignant mesothelioma. Journal of Clinical Oncology 2004; 22 (14 (S)).

49. Mathy A, Baas P, et al. Limited efficacy of imatinib mesylate in malignant mesothelioma: a phase II trial. Lung Cancer 2005; 50: 83-6.

50. Kindler HLV, NJ, Chien K, Stadler WM, Karczmar G, Heimann R, Vokes EE. SU 5416 in Malignant Mesothelioma: A University of Chicago Phase II Consortium Study. 2001 ASCO Meeting. Proc Am Soc Clin Oncol 2001; 20: 2001 (abstr 1359).

51. Pass HI, Brewer GJ, et al. A phase II trial of tetra- 
thiomolybdate after surgery for malignant mesothelioma: final results. Ann Thorac Surg 2008; 86: 383-9; discussion 390.

52. Bolden JE, Peart MJ, et al. Anticancer activities of histone deacetylase inhibitors. Nat Rev Drug Discov 2006; 5: 769-84.

53. Candelaria M, Gallardo-Rincon D, et al. A phase II study of epigenetic therapy with hydralazine and magnesium valproate to overcome chemotherapy resistance in refractory solid tumors. Ann Oncol 2007; 18: 1529-38.

54. Crisanti MC, Wallace AF, et al. The HDAC inhibitor panobinostat (LBH589) inhibits mesothelioma and lung cancer cells in vitro and in vivo with particular efficacy for small cell lung cancer. Mol Cancer Ther 2009; 8: 2221-31.

55. Ramalingam SS, Belani CP, et al. Phase II study of belinostat (PXD101), a histone deacetylase inhibitor, for second line therapy of advanced malignant pleural mesothelioma. J Thorac Oncol 2009; 4: 97-101.

56. Kelly WK, O'Connor OA, et al. Phase I study of an oral histone deacetylase inhibitor, suberoylanilide hydroxamic acid, in patients with advanced cancer. J Clin Oncol 2005; 23: 3923-31.

57. Scherpereel A, Berghmans T, Lafitte JJ, et al. for the European Lung Cancer Working Party (ELCWP). Valproate-doxorubicin: promising therapy for progressing mesothelioma. A phase II study. Eur Respir J 2010 Jun 7. [Epub ahead of print]

58. Sterman DH, Recio A, et al. A phase I clinical trial of single-dose intrapleural IFN-beta gene transfer for malignant pleural mesothelioma and metastatic pleural ef- fusions: high rate of antitumor immune responses. Clin Cancer Res 2007; 13: 4456-66.

59. Sterman DH, Recio A, et al. A phase I trial of repeated intrapleural adenoviral-mediated interferon-beta gene transfer for mesothelioma and metastatic pleural effusions. Mol Ther 18: 852-60.

60. Odaka M, Sterman DH, et al. Eradication of intraperitoneal and distant tumor by adenovirus-mediated interferon-beta gene therapy is attributable to induction of systemic immunity. Cancer Res 2001; 61: 6201-12.

61. Hopkins-Donaldson S, Cathomas R, et al. Induction of apoptosis and chemosensitization of mesothelioma cells by $\mathrm{Bcl}-2$ and $\mathrm{Bcl}-\mathrm{xL}$ antisense treatment. Int $J$ Cancer 2003; 106: 160-6.

62. Yang CT, You L, et al. A comparison analysis of antitumor efficacy of adenoviral gene replacement therapy (p14ARF and p16INK4A) in human mesothelioma cells. Anticancer Res 2003; 23: 33-8.

63. Hegmans JP, Hemmes A, et al. Immunotherapy of murine malignant mesothelioma using tumor lysatepulsed dendritic cells. Am J Respir Crit Care Med 2005; 171: 1168-77

64. Gregoire M, Ligeza-Poisson C, et al. Anti-cancer therapy using dendritic cells and apoptotic tumour cells: pre-clinical data in human mesothelioma and acute myeloid leukaemia. Vaccine 2003; 21: 791-4.

65. Hegmans JP, Veltman JD, Lambers ME, et al. Consolidative Dendritic Cell-Based Immunotherapy Elicits Cytotoxicity Against Malignant Mesothelioma. Am J Respir Crit Care Med 2010 Jun 15; 181: 1383-90. Epub 2010 Feb 18.

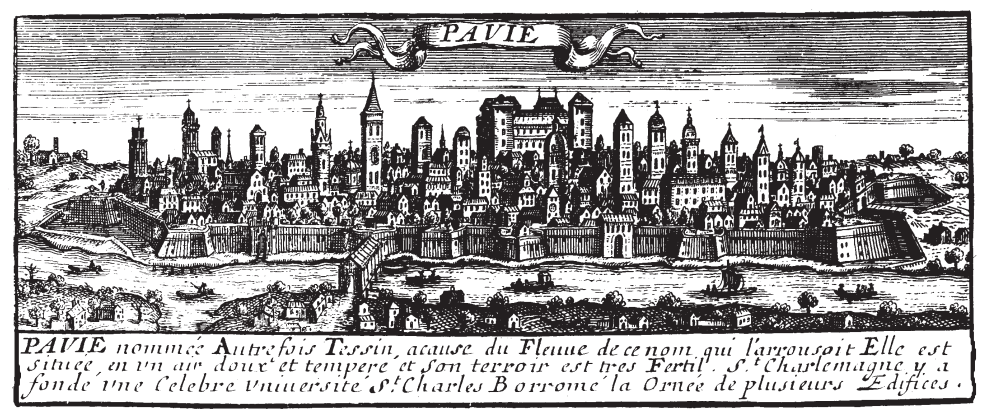

